\title{
ON THE EXISTENCE OF PROJECTIVE AFFINE MOTION IN A $W$-RECURRENT FINSLER SPACE
}

\author{
A. KUMAR, H. S. SHULKA AND R. P. TRIPATHI
}

\begin{abstract}
The paper is devoted to study the properties of a $W-R F_{n}$ space admitting an infinitesimal point transformation $\bar{x}^{i}=x^{i}+v^{i}(x) d t$ which satisfies the condition $L_{v} \lambda_{s}=0$
\end{abstract}

\section{Introduction}

Let us consider an $n$-dimensional affinely connected Finsler space $F_{n}[1]^{1}$ equipped with $2 n$ line elements $\left(x^{i}, \dot{x}^{i}\right)$ and a fundamental metric function $F(x, \dot{x})$ positively homogeneous of degree one in its directional argument. The fundamental metric tensor $g_{i j}(x, \dot{x}) \stackrel{\operatorname{def}_{1}}{=} \dot{\partial}_{i} \dot{\partial}_{j} F^{2}(x, \dot{x})^{2}$ of the space is symmetric in its indices $i$ and $j$. Let $T_{j}^{i}(x, \dot{x})$ be any tensor field depending on both the positional and directional arguments. The covariant derivative of $T_{j}^{i}(x, \dot{x})$ with respect to $x^{k}$ in the sense of Berwald is given by

$$
T_{j(k)}^{i}=\partial_{k} T_{j}^{i}-\dot{\partial}_{h} T_{j}^{i} G_{k}^{h}+T_{j}^{h} G_{h k}^{i}-T_{h}^{i} G_{j k}^{h}
$$

where $G_{j k}^{i}(x, \dot{x})$ are Berwald's connection coefficients and satisfy the following relations:
a) $\dot{\partial}_{h} G_{j k}^{i}=G_{h j k}^{i}$
b) $G_{h j k}^{i} \dot{x}^{h}=0$ and
c) $G_{h k}^{i}=G_{k h}^{i}$

The following commutation formulae involving the Berwald's covariant derivatives are given by

$$
\begin{aligned}
\dot{\partial}_{h} T_{j(k)}^{i}-\left(\dot{\partial}_{h} T_{j}^{i}\right)_{(k)} & =T_{j}^{s} G_{s h k}^{i}-T_{s}^{i} G_{j h k}^{s}, \\
{ }^{3} 2 T_{j[(h)(k)]}^{i} & =-\dot{\partial}_{r} T_{j}^{i} H_{h k}^{r}+T_{j}^{s} H_{s h k}^{i}-T_{s}^{i} H_{j h k}^{s}
\end{aligned}
$$

Received February 12, 1998; revised October 30, 1999.

1991 Mathematics Subject Classification. 53C.

Key words and phrases. Finsler space, projective motion, recurrent, Lie-derivative, infinitesimal point transformation, connection coefficients.

${ }^{1}$ The numbers in square brackets refer to the references at the end of the paper.

${ }^{2} \dot{\partial}_{i} \equiv \partial / \partial \dot{x}^{i}$ and $\partial_{i} \equiv \partial / \partial x_{i}$

${ }^{3} 2 A_{[h k]}=A_{h k}-A_{k h}$ 
where

$$
H_{h j k}^{i}(x, \dot{x}) \stackrel{\text { def }}{=} 2\left\{\partial_{[k} G_{j] h}^{i}+G_{h[j}^{r} G_{k] r}^{i}+G_{r h[k}^{i} G_{j]}^{r}\right\}
$$

are Berwald's curvature tensor field and satisfies the following relations:
a) $H_{h j k}^{i}=-H_{h k j}^{i}$,
b) $H_{h j k}^{i} \dot{x}^{h}=H_{j k}^{i}$,
c) $H_{r h j}^{r}=2 H_{[h j]}$
d) $H_{h j r}^{r}=H_{h j}$,
e) $H_{i}^{i}=(n-1) H$ and
f) $\dot{x}^{j} H_{j k}^{i}=H_{k}^{i}$

The projective deviation tensor field $W_{h j k}^{i}(x, \dot{x})$ of the space is given by

$$
W_{h j k}^{i}(x, \dot{x})=H_{h j k}^{i}+\frac{1}{(n+1)}\left\{\delta_{h}^{i} H_{r k j}^{r}+\dot{x}^{i} \dot{\partial}_{h} H_{r k j}^{r}+2 \delta_{[j}^{i}\left(H_{(h) k]}+\dot{\partial}_{k]} \dot{\partial}_{h} H\right)\right\}
$$

which satisfies the following identity:

$$
W_{h j k(\ell)}^{i}+W_{h k \ell(j)}^{i}+W_{h \ell j(k)}^{i}=0
$$

If the projective deviation tensor field $W_{h j k}^{i}(x, \dot{x})$ satisfies the condition

$$
W_{h j k(s)}^{i}=\lambda_{s} W_{h j k}^{i}
$$

where $\lambda_{s}(x)$ means a non-zero covariant recurrence vector, the space is called a $W$ recurrent Finsler space or an $W-R F_{n}$ space.

Let us consider an infinitesimal point transformation

$$
\bar{x}^{i}=x^{i}+v^{i}(x) d t
$$

where $v^{i}(x)$ is any vector field and $d t$ is an infinitesimal point constant. The above transformation which is considered at each point in the space is called a projective affine motion, when and only when

$$
L_{v} G_{j k}^{i}=0
$$

where $L_{v}$ denotes the well known Lie-derivative with respect to (1.10). The Lie-derivatives of the tensor field $T_{j}^{i}(x, \dot{x})$ and connection coefficient $G_{j k}^{i}(x, \dot{x})$ in view of (1.10) and the Berwald's covariant derivative are given by [2]

$$
L_{v} T_{j}^{i}=T_{j(h)}^{i} v^{h}+T_{h}^{i} v_{(\dot{j})}^{h}-T_{j}^{h} v_{(h)}^{i}+\dot{\partial}_{h} T_{j}^{i} v_{(s)}^{h} \dot{x}^{s}
$$

and

$$
L_{v} G_{j k}^{i}=v_{(j)(k)}^{i}+H_{j k h}^{i} v^{h}+G_{s j k}^{i} v_{(r)}^{s} \dot{x}^{r}
$$

respectively.

We have the following commutation formulae:

$$
\begin{aligned}
& L_{v}\left(\dot{\partial}_{\ell} T_{j}^{i}\right)-\dot{\partial}_{\ell} L_{v} T_{j}^{i}=0 \\
& \left(L_{v} G_{j h}^{i}\right)_{(k)}-\left(L_{v} G_{k h}^{i}\right)_{(j)}=L_{v} H_{h j k}^{i}+2 \dot{x}^{s} G_{r h[j}^{i} L_{v} G_{k] s}^{r}
\end{aligned}
$$


and

$$
\left(L_{v} T_{j k(m)}^{i}\right)-\left(L_{v} T_{j k}^{i}\right)_{(m)}=T_{j k}^{s} L_{v} G_{s m}^{i}-T_{s k}^{i} L_{v} G_{j m}^{s}-T_{j s}^{i} L_{v} G_{k m}^{s}-\dot{\partial}_{s} T_{j k}^{i} L_{v} G_{r m}^{s} \dot{x}^{r}
$$

Hence, for an infinitesimal projective affine motion the last relation shows that the two operators $L_{v}$ and $(k)$ are commutative with each other.

With the help of the equation (1.11) and (1.15), we get

$$
L_{v} H_{h j k}^{i}=0
$$

In view of the equation (1.6) and the fact that the operations of contraction and Lie-derivation are commutative the above relation yields
a) $L_{v} H_{r j k}^{r}=0$,
b) $L_{v} H_{j k}=0$ and
c) $L_{v} H=0$

Taking the Lie-derivative of the each side of (1.7) and using the equations (1.14), (1.17) and (1.18), we obtain

$$
L_{v} W_{h j k}^{i}=0
$$

Applying $L_{v}$ to both sides of (1.9) and using the equations (1.11), (1.16) and (1.19), we have

$$
\left(L_{v} \lambda_{s}\right) W_{h j k}^{i}=0
$$

Since the space is not an isotropic (i.e. $W_{h j k}^{i} \neq 0$ ), we have

$$
L_{v} \lambda_{s}=0
$$

i.e. the recurrence vector $\lambda_{s}$ of the space must be Lie-invariant one.

In what follows, we shall study a $W-R F n$ space admitting an infinitesimal transformation $\bar{x}^{i}=x^{i}+v^{i}(x) d t$ which satisfies (1.21). We shall call such a restricted space, for brevity, as $S-W R$ Fn space.

\section{The Vanishing of $L_{v} W_{h j k}^{i}(x, \dot{x})$}

First of all here we shall prove the following lemma:

Lemma 2.1. In an $S-W R$ Fn space if the recurrence vector $\lambda_{s}$ is a gradient one, we have $\lambda_{s} v^{s}=$ const.

Proof. For brevity, let us put

$$
\delta=\lambda_{s} v^{s}
$$

Then, with the help of the equations (1.12) and (1.21), we have

$$
L_{v} \lambda_{s}=\lambda_{s(m)} v^{m}+\lambda_{m} v_{(s)}^{m}=0
$$


By virtue of the assumption $\lambda_{s(m)}=\lambda_{m(s)}$ the above equation reduces to

$$
\delta_{(m)}=0
$$

which completes the proof.

In view of the basic condition (1.12), the Lie-derivative of $W_{h j k}^{i}(x, \dot{x})$ is given by

$$
L_{v} W_{h j k}^{i}=W_{h j k(s)}^{i} v^{s}+W_{s j k}^{i} v_{(h)}^{s}+W_{h s k}^{i} v_{(j)}^{s}+W_{h j s}^{i} v_{(k)}^{s}-W_{h j k}^{s} v_{(s)}^{i}+\dot{\partial}_{s} W_{h j k}^{i} v_{(r)}^{s} \dot{x}^{r}
$$

which by virtue of the equation (1.9) and (2.1) reduces to

$$
L_{v} W_{h j k}^{i}=\delta W_{h j_{k}}^{i}+W_{s j k}^{i} v_{(h)}^{s}+W_{h s k}^{i} v_{(j)}^{s}+W_{h j s}^{i} v_{(k)}^{s}-W_{h j k}^{s} v_{(s)}^{i}+\dot{\partial}_{s} W_{h j k}^{i} v_{(r)}^{s} \dot{x}^{r}
$$

Introducing the commutation formula (1.4) to the tensor field $W_{h j k}^{i}(x, \dot{x})$, we get

$$
2 W_{h \dot{j k}[(\ell)(m)]}^{i}=-\dot{\partial}_{r} W_{h j k}^{i} H_{s \ell m}^{r} \dot{x}^{s}+W_{h j k}^{s} H_{s \ell m}^{i}-W_{s j k}^{i} H_{h \ell m}^{s}-W_{h s k}^{i} H_{j \ell m}^{s}-W_{h j s}^{i} H_{k \ell m}^{s} .
$$

In view of the definition (1.9), the above relation reduces to

$$
\left(\delta_{\ell(m)}-\delta_{m(\ell)}\right) W_{h \dot{j} k}^{i}=-\dot{\partial}_{r} W_{h \dot{j} k}^{i} H_{s \ell m}^{r} \dot{x}^{s}+W_{h \dot{j} k}^{s} H_{s \ell m}^{i}-W_{s j k}^{i} H_{h \ell m}^{s}-W_{h s k}^{i} H_{j \ell m}^{s}-W_{h j s}^{i} H_{k \ell m}^{s} .
$$

Next, let us assume that $\delta_{m} \neq$ const. Then, with the help of the Lemma (2.1), we get

$$
N_{\ell m}(x) \stackrel{d e f}{=}\left(\delta_{\ell(m)}-\delta_{m(\ell)}\right) \neq 0
$$

Let us take

$$
v_{(h)}^{i}=H_{h j k}^{i} q^{j k}
$$

for a suitable non-symmetric tensor $q^{j k}$, then multiplying (2.7) by $q^{l m}$ and summing over 1 and $m$, we obtain

$$
N_{\ell m} q^{\ell m} W_{h j k}^{i}=-\dot{\partial}_{r} W_{h j k}^{i} v_{(s)}^{r} \dot{x}^{s}+W_{h j k}^{s} v_{(s)}^{i}-W_{s j k}^{i} v_{(h)}^{s}-W_{h s k}^{i} v_{(j)}^{s}-W_{h j s}^{i} v_{(k)}^{s}
$$

Comparing the last equation with (2.5), we get

$$
L_{v} W_{h j k}^{i}=\left(\delta-q^{\ell m} N_{\ell m}\right) W_{h j k}^{i}
$$

The above equation shows that $L_{v} W_{h j k}^{i}$ vanishes when and only $\delta=q^{l m} N_{\ell m}$.

For $\delta \neq$ const. and $N_{\ell m} \neq 0$, from (2.5) and (2.7), we can construct the following identity

$$
\begin{aligned}
N_{\ell m} L_{v} W_{h j k}^{i}= & W_{h j k}^{s}\left(\delta H_{s \ell m}^{i}-N_{\ell m} v_{(s)}^{i}\right)-W_{s j k}^{i}\left(\delta H_{h \ell m}^{s}-N_{\ell m} v_{(h)}^{s}\right) \\
& -W_{h s k}^{i}\left(\delta H_{j \ell m}^{s}-N_{\ell m} v_{(j)}^{s}\right)-W_{h j s}^{i}\left(\delta H_{k \ell m}^{s}-N_{\ell m} v_{(k)}^{s}\right)
\end{aligned}
$$


Thus, for $L_{v} W_{h j k}^{i}=0$, the above equation yields [6]:

$$
\delta H_{s \ell m}^{i}=N_{\ell m} v_{(s)}^{i}
$$

where $v^{i}$ does not mean a parallel vector.

We define

Definition 2.1. A $S$-WR Fn space satisfying $\lambda_{m} v^{m} \neq$ const. is called a special one of the first kind.

Next, let us go back to the case, $\lambda_{m} v^{m}=$ const. of the foregoing Lemma (2.1). Then, (2.7) is replaced by

$$
-\dot{\partial}_{r} W_{h j k}^{i} H_{s \ell m}^{r} \dot{x}^{s}+W_{h j k}^{s} H_{s \ell m}^{i}-W_{s j k}^{i} H_{h \ell m}^{s}-W_{h s k}^{i} H_{j \ell m}^{s}-W_{h j s}^{i} H_{k \ell m}^{s}=0
$$

Transvecting it by $q^{l m}$ and remembering the equation (2.9) we get

$$
-\dot{\partial}_{r} W_{h j k}^{i} v_{(s)}^{r} \dot{x}^{s}+W_{h j k}^{s} v_{(s)}^{i}-W_{s j k}^{i} v_{(h)}^{s}-W_{h s k}^{i} v_{(j)}^{s}-W_{h j s}^{i} v_{(k)}^{s}=0
$$

Substituting the above equation into the right hand side of (2.5), we obtain

$$
L_{v} W_{h j k}^{i}=\delta W_{h j k}^{i}
$$

Therefore, when the arbitrary constant $\delta$ vanishes, we have

$$
L_{v} W_{h j k}^{i}=0
$$

We put the

Definition. An $S$-WR Fn space is called a special one of the second kind when $\lambda_{m} v^{m}=$ const. holds good.

Then, summarizing the above results, we have the following theorems.

Theorem 2.1. In a special $S-W R$ Fn space of the first kind, if the space has the resolved curvature $H_{h j k}^{i}$ of the form (2.13), $L_{v} W_{h j k}^{i}=0$ holds good.

Theorem 2.2. In a special $S-W R$ Fn space of the second kind, if the arbitrary constant $\lambda_{m} v^{m}$ vanishes, we have $L_{v} W_{h j k}^{i}=0$. From the last theorem, if $\lambda_{m}=0$, then with the help of the equation (1.9), we have

$$
W_{h i k(r)}^{i}=0
$$

Thus, we have

Corollary 2.1. In a symmetric Finsler space, $L_{v} W_{h j k}^{i}=0$, is satisfied identically. 


\section{Complete Condition}

In this section we shall find the necessary and sufficient condition for (2.13). From the assumption (1.21), we have

$$
L_{v} \lambda_{m}=\lambda_{m(s)} v^{s}+\left(\lambda_{m} v^{s}\right)_{(m)}-\lambda_{s(m)} v^{s}=0
$$

By virtue of (2.1) and (2.8), the last equation reduces to

$$
\delta_{(m)}+N_{m s} v^{s}=0
$$

In view of the equation (1.12), the Lie-derivative of $N_{l m}(x)$ is given by

$$
L_{v} N_{\ell m}=N_{\ell m(s)} v^{s}+N_{s m} v_{(\ell)}^{s}+N_{\ell s} v_{(m)}^{s}
$$

Remembering the commutation formula (1.16), we have

$$
L_{v}\left(\lambda_{m(s)}-\left(L_{v} \lambda_{m}\right)_{(s)}=-\lambda_{r} L_{v} G_{m s}^{r}\right.
$$

With the help of the equation $(1.2 \mathrm{c}),(1.21)$ and (2.8), the above relation reduces to

$$
L_{v} N_{s m}=0 .
$$

Differentiating (2.7), convariantly with respect to $x^{n}$ and using the equations (1.3), (1.9), (2.7) and (2.8), we obtain

$$
N_{\ell m(n)} W_{h j k}^{i}=\lambda_{n} W_{h j k}^{i} N_{\ell m}+H_{a \ell m}^{r} \dot{x}^{a}\left(W_{h j k}^{s} G_{s r n}^{i}-W_{s j k}^{i} G_{h r n}^{s}-W_{h s k}^{i} G_{j r n}^{s}-W_{h j s}^{i} G_{k r n}^{s}\right)
$$

Transvecting it by $\dot{x}^{n}$ and noting the equations (1.2b), we get after a little simplification:

$$
N_{\ell m(n)}=\lambda_{n} N_{\ell m}
$$

Thus, by virtue of the equations (3.3), (3.5) and (3.7), we get

$$
\delta N_{\ell m}+N_{s m} v_{(\ell)}^{s}+N_{\ell s} v_{(m)}^{s}=0
$$

Next, from the equation (3.2), we have

$$
\delta_{(m)(n)}-\delta_{(n)(m)}=-\left(N_{m s} v^{s}\right)_{(n)}+\left(N_{n s} v^{s}\right)_{(m)}
$$

being $\delta$ a non-constant scalar function, the above equation reduces to

$$
N_{m s} v_{(n)}^{s}-N_{s n} v_{(m)}^{s}=-\lambda_{n} N_{m s} v^{s}+\lambda_{m} N_{n s} v^{s}
$$

where, we have used (3.7) and $N_{m s}=-N_{s m}$. Substituting the last equation into the left hand side of (3.8), we get

$$
\delta N_{m n}=-\lambda_{n} \delta_{(m)}+\lambda_{m} \delta_{(n)}
$$


In an affinely connected space the identify (1.8) reduces to

$$
W_{h j k(\ell)}^{i}+W_{h k \ell(j)}^{i}+W_{h \ell j(k)}^{i}=0
$$

which in view of the definition (1.9) reduces to

$$
\delta W_{h j k}^{i}=\lambda_{k} W_{h j \ell}^{i} v^{\ell}-\lambda_{j} W_{h k s}^{i} v^{s}
$$

Where, we have used (2.1) and $W_{h j k}^{i}=-W_{h k j}^{i}$. Hence, from (3.11) and (3.13), we can make the following identity:

$$
\delta\left(\delta W_{h j k}^{i}-N_{j k} v_{(h)}^{s}\right)=\lambda_{k}\left(\delta W_{h j s}^{i} v^{s}+\delta_{(\dot{j})} v_{(h)}^{i}\right)-\lambda_{j}\left(\delta W_{h k s}^{i} v^{s}+\delta_{(k)} v_{(h)}^{i}\right)
$$

Consequently (2.13) follows when and only when, we have

$$
\delta w_{h j s}^{i} v^{s}+\delta_{(\dot{j})} v_{(h)}^{i}=\lambda_{j} Q_{h}^{i}
$$

where $Q_{h}^{i}$ means a suitable tensor. Transvecting the above equation by $v^{j}$ and summing over $j$ by virtue of $W_{h j k}^{i} v^{j} v^{k}=0$ and $\delta_{(j)} v^{j}=0$ derived from (3.2), we get

$$
\delta Q_{h}^{i}=0
$$

where we have used (2.1). Since $\delta \neq 0$, therefore, the last relation yields $Q_{h}^{i}=0$. Thus, from (3.15), we have

$$
W_{h j s}^{i} v^{s}+\delta_{j} v_{(h)}^{i}=0, \quad\left(\delta_{j} \equiv \delta_{(j)} / \delta\right)
$$

In this way, we have the

Theorem 3.1. In order that we have (2.13), (3.17) is necessary and sufficient.

Now the equation (3.17) suggests the concrete form of the tensor $q^{l m}$ used in the first half of $\S 2$. In fact if $\delta_{m} \neq 0$ there exists a suitable vector $\rho^{m}$ such that

$$
\delta_{m} \rho^{m}=1
$$

Then transvecting (3.17) by $\rho^{j}$ and noting the above relation, we get

$$
v_{(h)}^{i}=\delta W_{h s j}^{i} v^{s} \rho^{j}
$$

If, we introduce

$$
q^{l m}=v^{l} \rho^{m}
$$

then $N_{l m} q^{l m}=N_{l m} v^{l} \rho^{m}=\delta_{(m)} \rho^{m}=\delta \cdot \delta_{m} \rho^{m}=\delta$ That is from (3.17) and (2.13), we have

$$
\delta=N_{l m} q^{l m}
$$

straightway. Therefore, we can take (3.20) concretely. Hence in order to have the concrete form of $q^{l m},(3.17)$ should be taken as a basic condition in our theory. If this is done, we are able to have (2.13) always, so $L_{v} W_{h j k}^{i}=0$ holds good.

Thus, we have

Theorem 3.2. If we introduce $v_{(h)}^{i}$ by $(3.17), L_{v} w_{h j k}^{i}=0$ is satisfied identically. 


\section{References}

[1] H. Rund, The differential geometry of Finsler Spaces, Springer-Verlag, Berlin, 1959.

[2] K. Yano, The theory of Lie-derivatives and its applications P. Noordhoff, Groningen, 1957.

[3] Y. C. Wong, A class of non-Riemannian $K *$-space, Proc. of the London Math. Soc., 3(1958), 118-128.

[4] M. S. Knebelman, On the equations of motions in a Riemann space, Bull. Amer. Math. Soc., 51(1945), 682-685.

[5] W. Slebodzinski, Sur les transformations isomorphiques dune varie'te a' connexion affine, Prace Mat. Fiz., 39(1932), 55-62.

[6] K. Takano, On the existence of affine motion in a space with recurrent curvature tensor, Tensor, N. S., 10(1966), 68-73.

Department of Mathematics, DDU Gorakhpur University, Gorakhpur-273009, India. 